\title{
Charge Storage in Conducting Polymers: Solitons, Polarons, and Bipolarons
}

\author{
A. J. HeEger \\ Institute for Polymers and Organic Solids, \\ University of California at Santa Barbara \\ Santa Barbara, California 93106, U.S.A.
}

(Received August 20, 1984)

\begin{abstract}
The results of a series of experiments demonstrate that solitons are the important excitations in trans- $(\mathrm{CH})_{x}$ and that the properties of these nonlinear excitations can be directly studied during photoexcitation or after doping. The importance of these concepts in the more general context of conducting polymers is addressed. Although the two-fold degenerate ground state of trans- $(\mathrm{CH})_{x}$ is quite special, the relevant concepts have been generalized to confined soliton pairs (bipolarons). Experimental results which demonstrate electron-hole symmetry and weak confinement in poly(thiophene) make this polyheterocycle a nearly ideal example of a model system in which the ground state degeneracy has been lifted. In the dilute doping regime, in-situ absorption spectroscopy data (during electrochemical doping) are in detailed agreement with charge storage via bipolarons with confinement parameter $\gamma \simeq 0.1-0.2$. These results on polythiophene demonstrate that a quantitative fundamental understanding is possible even for relatively complex systems.

KEY WORDS Poly(acetylene)/Poly(thiophene)/Charge Storage / Conducting Polymers /
\end{abstract}

In traditional three-dimensional semicounductors, the four-fold (or six-fold, etc.) coordination of each atom to its neighbor through covalent bonds leads to a rigid structure. In such systems, therefore, the electronic excitations can usually be considered in the context of this rigid structure leading to the conventional concepts of electrons and holes as the dominant excitations. The situation in semiconductor polymers is quite different; the two-fold coordination makes these systems generally more susceptible to structural distortion. As a result, the dominant "electronic" excitations are inherently coupled to chain distortions. Thus, quite generally, one anticipates that solitons, polarons, and bipolarons will be the excitations of major importance in this class of one-dimensional polymer semiconductors. We illustrate these concepts in this paper through a summary of experimental results on two systems:

1) Polyacetylene, $(\mathrm{CH})_{x}$; the degenerate ground state leads to solitons as the important excitations and the dominant charge storage species.

2) Poly(thiophene); the ground state degeneracy is lifted so that polarons and bipolarons are the important excitations with charge storage in bipolarons.

As shown in Figure 1, trans- $(\mathrm{CH})_{x}$ is a two-fold degenerate Peierls insulator which allows for the possibility of non-linear excitations in the form of soliton-like bond-alternation domain walls, each with an associated electronic state at the center of the energy gap. ${ }^{1-5}$ For polythiophene, on the other hand, the two structures sketched in Figure 2 are not energetically equivalent. Poly(thiophene) (PT) can be viewed as an $\mathrm{sp}^{2} \mathrm{p}_{\mathrm{z}}$ carbon chain in a structure somewhat analogous to that of $c i s-(\mathrm{CH})_{x}$, but stabilized in that structure by the sulfur, which covalently bonds to neighboring carbons to form the heterocycle.

In this paper, we briefly summarize the results of the series of experiments which demonstrate that solitons are important excitations in trans- $(\mathrm{CH})_{x}$, and that the properties of these nonlinear excitations can be directly studied through measurements on trans- $(\mathrm{CH})_{x}$ samples either during photoexcitation or after doping. Having established the 


\section{A. J. HEEGER}

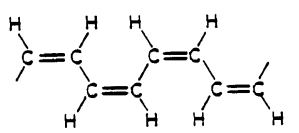

CIS
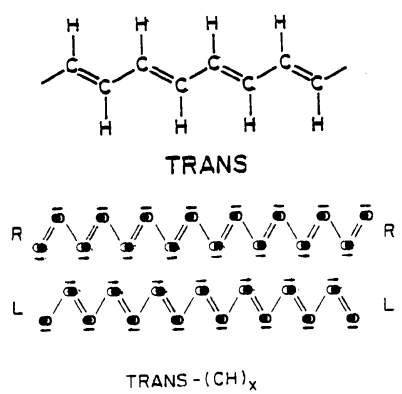

Figure 1. a) The chemical structures of $c i s-(\mathrm{CH})_{x}$ and trans- $(\mathrm{CH})_{x}$. b) The two degenerate ground state structures of trans- $(\mathrm{CH})_{x}$. The atomic distortions are indicated by the arrows.

(a)

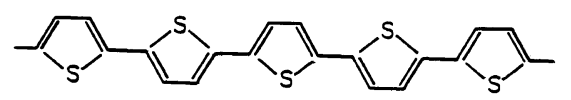

(b)

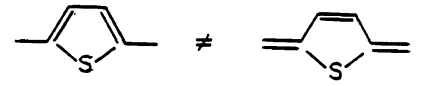

Figure 2. a) Chemical structure diagram of poly(thiophene). b) Two inequivalent structures for the thiophene heterocycle in poly(thiophene).

relevance of these coupled electronic-lattice excitations in trans- $(\mathrm{CH})_{x}$, the importance of these concepts in the more general context of conducting polymers is addressed. Although the two-fold degenerate ground state of trans- $(\mathrm{CH})_{x}$ is quite special, the relevant concepts can be generalized to confined soliton pairs (bipolarons) and applied to a wide variety of conjugated polymers in which the ground state degeneracy is not present. Experimental results which demonstrate electron-hole symmetry and weak confinement in poly(thiophene) make this polyheterocycle a nearly ideal example of a model system in which the ground state degeneracy has been lifted.

\section{SOLITONS AND BIPOLARONS: SOME CONCEPTS}

The soliton in $(\mathrm{CH})_{x}$ is a topological kink in the electron-lattice system; a bond alternation domain wall connecting the two phases with opposite bond

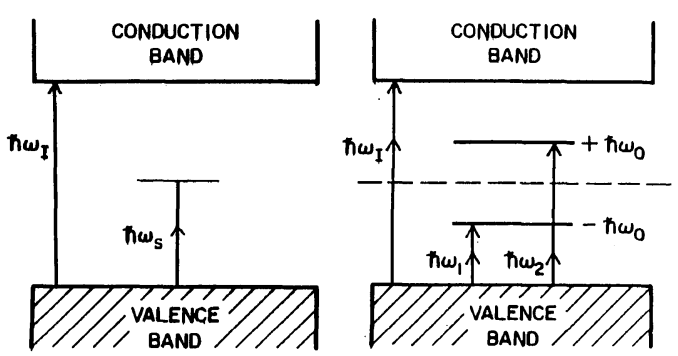

Figure 3. a) Energy level diagram for a charged (positive) soliton in trans- $(\mathrm{CH})_{x}$. The allowed transitions are at $\hbar \omega_{\mathrm{I}}=2 \Delta$ (interband) and $\hbar \omega_{\mathrm{S}}=\Delta$ (soliton midgap transition). b) Energy level diagram for a charged (positive) bipolaron. Because of the splitting of the levels there are three possible transitions in addition to $\hbar \omega_{1}$.

alternation. Since there is translational symmetry (the kink can be anywhere) and since the mass is small, the soliton should be mobile. The competition of elastic and condensation energies spreads the domain wall over a region of about $12-14 a$, where $a$ is the $\mathrm{C}-\mathrm{C}$ distance along the $(\mathrm{CH})_{x}$ chain. Although single soliton defects can exist on imperfect chains (and have been studied extensively), ${ }^{6,7}$ intrinsic excitations, either photoproduced or doping induced, must occur in the form of soliton-antisoliton $(\mathbf{S}-\overline{\mathbf{S}})$ pairs.

Associated with the structural kink is a localized electronic state with energy at mid-gap (see Figure 3a). This electronic state is a solution of the Schrödinger equation in the presence of the structural domain wall and can therefore accommodate 0,1 , or 2 electrons. ${ }^{1-5}$ The neutral soliton has one electron in the mid-gap state; the positive and negative charged solitons have zero or two electrons, respectively, in the mid-gap state. Consequently, the spin-charge relations of solitons are reversed; charged solitons are non-magnetic, whereas a neutral soliton has spin $1 / 2$. The reversed spincharge relationship for solitons in trans- $(\mathrm{CH})_{x}$ is a direct manifestation of charge fractionalization discovered in the mathematical analysis of spinless fermion systems. ${ }^{8}$ In trans- $(\mathrm{CH})_{x}$, the localized midgap state of the soliton derives from one-half a state removed from the occupied valence band and onehalf a state from the empty conduction band for each sign of spin. Thus, although the charge fractionalization is masked by the spin degeneracy in trans- $(\mathrm{CH})_{x}$, the resulting reversed spin-charge relation has the same physical origin. 

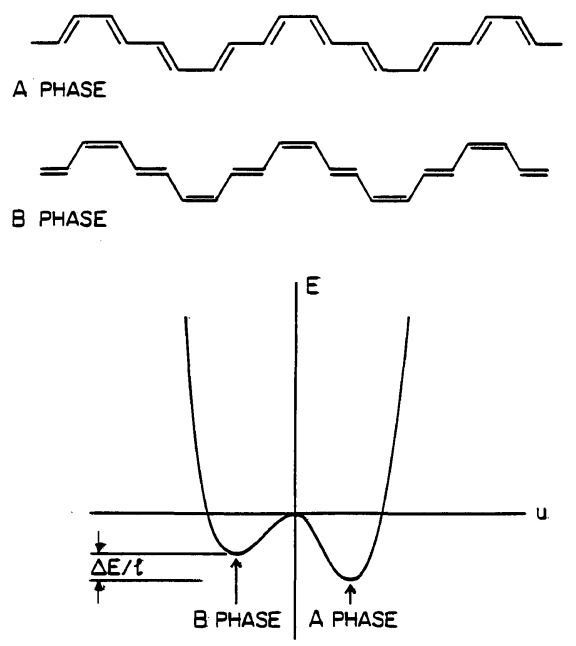

Figure 4. Schematic diagram of poly(thiophene) backbone structure leaving out the sulfur atoms (see Fig. 2). The two configurations (A phase and B phase) are nearly (but not precisely) degenerate as shown in the diagram at the bottom of the figure where we plot energy versus the distortion parameter, $u$ ( $u=0$ when the bond lengths are equal).

Theoretical analysis ${ }^{2-5}$ has demonstrated that a charged soliton represents the lower energy configuration for an excess charge on a trans $-(\mathrm{CH})_{x}$ chain; i.e., $E_{\mathrm{S}}<\Delta$ where $E_{\mathrm{S}}$ is the energy for creation of a soliton and $\Delta$ is the energy for creation of an electron or hole, $\Delta=E_{\mathrm{g}} / 2$. Both numerical calculations using a discrete lattice model, ${ }^{2}$ and analytical results for a continuum model ${ }^{4,5}$ indicate that neglecting electron-electron Coulomb interaction)

$$
2 E_{\mathrm{S}}=(4 / \pi) \Delta
$$

Charged solitons are therefore formed by electron transfer onto or off of the polymer chains when doping with electron donors or acceptors.

An analogy can be constructed ${ }^{9}$ between PT and $(\mathrm{CH})_{x}$ as shown in the diagrams of Figure 4 where we redraw the polythiophene backbone structure, purposely leaving out the sulfur heteroatom. The resulting structure is that of an $\mathrm{sp}^{2} \mathrm{p}_{z}$ polyene chain consisting of four carbon all-trans segments linked through a cis-like unit. In such a structure the ground state is not degenerate (as sketched in Figure 4). However, the energy difference per bond, $\Delta E / l$, might be expected to be small; i.e., greater than zero (as in trans- $(\mathrm{CH})_{x}$ ) but less than that of cis- $(\mathrm{CH})_{x}$. An obvious consequence of the lack of degeneracy is that the schematic PT structure of Figure 4 cannot support stable soliton excitations, ${ }^{1,5,10-12}$ since creating a soliton pair separated by a distance $d$ would cost energy $\sim d(\Delta E / l)$. This linear "confinement" energy leads to bipolarons as the lowest energy charge transfer configurations in such a chain with creation energy somewhat greater than $(4 / \pi) \Delta$ (the creation energy for a bipolaron goes to eq 1 in the limit of zero confinement). The corresponding energy level diagram (for a positive bipolaron) is sketched in Figure $3 \mathrm{~b}$. The two gap states are empty for a positive bipolaron (charge $2|e|)$ and filled for a negative bipolaron (charge $-2|e|$ ).

\section{SOLITONS IN POLYACETYLENE: SOME EXPERIMENTAL RESULTS}

\section{a) In-Situ Visible-IR Absorption Studies of trans- $(\mathrm{CH})_{x}$}

Electrochemical studies ${ }^{13,14}$ have allowed, for the first time, precise control of the doping process and quantitative measurement of the dopant concentration. The in-situ visible-IR absorption studies ${ }^{15}$ when combined with Electrochemical Voltage Spectroscopy ${ }^{16}$ (EVS) demonstrate that the charge is stored in the mid-gap states. Moreover, the EVS data give directly the energies for charge injection and removal. ${ }^{16}$ Analysis of these data yields a value for the soliton formation energy, $E_{\mathrm{S}}$, in good agreement with eq 1 .

The results ${ }^{15}$ of in-situ measurements (carried out during electrochemical doping) of the visible-IR absorption in trans- $(\mathrm{CH})_{x}$ are shown in Figure 5. As the doping proceeds, the mid-gap absorption appears, centered near $0.65-0.75 \mathrm{eV}$ with an intensity which increases monotonically in proportion to the dopant concentration. These spectroscopic features are independent of dopant species and independent of whether the doping is p-type (oxidation) or ntype (reduction). ${ }^{16 \mathrm{c}}$ Figure 6 shows optical absorption spectra for polyacetylene doped with different species $\left(\mathrm{Na}, \mathrm{Li}, \mathrm{Bu}_{4} \mathrm{~N}, \mathrm{ClO}_{4}\right)$ at $y \sim 1 \%$. The $\mathrm{Na}$ samples were chemically doped, the others electrochemically (using in-situ opto EVS technique). The similarity between these data proves conclusivety that the optical spectra of trans $-(\mathrm{CH})_{x}$ depends only on the properties of the doped polymer, and not on the particular dopant species.

The mid-gap optical absorption provides direct 


\section{A. J. HeEgER}

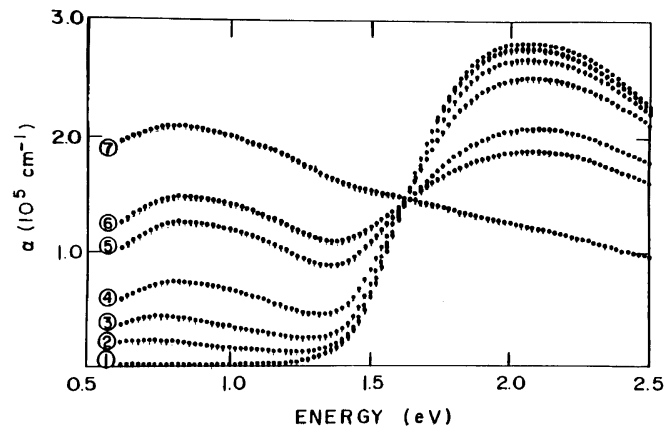

Figure 5. In-situ absorption curves for trans- $(\mathrm{CH})_{x}$, during electrochemical doping with $\left(\mathrm{ClO}_{4}\right)^{-}$. The applied voltages ( $v s . \mathrm{Li})$ and corresponding dopant concentrations are as follows: Curve 1, 2.2 volts $(y=0)$; curve $2,3.28$ volts $(y=0.03)$; curve $3,3.37$ volts $(y=$ $0.0065)$; curve $4,3.46$ volts ( $y=0.012$ ); curve $5,3.57$ volts $(y=0.027)$; curve $6,3.64$ volts $(y=0.047)$; curve $7,3.73$ volts $(y=0.078)$.

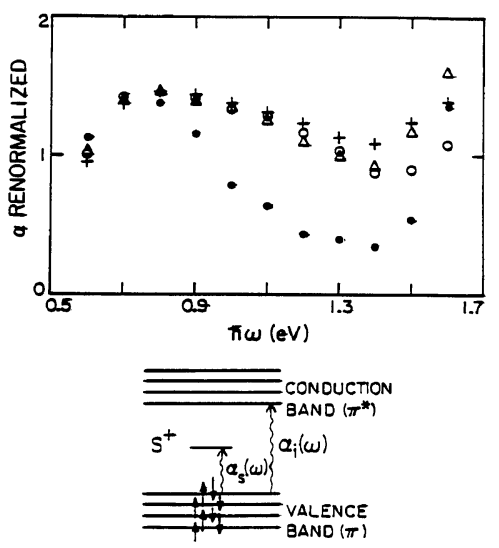

Figure 6. Near IR absorption spectra showing the mid-gap transition for trans- $(\mathrm{CH})_{x}$ doped with $\mathrm{Na}^{+}=$ $\mathrm{O}, \mathrm{Li}^{+}=\triangle, \mathrm{Bu}_{4} \mathrm{~N}^{+}=\mathbf{O}$ and $\mathrm{ClO}_{4}{ }^{-}=+$(see Kaufman et al. $\left.{ }^{160}\right)$.

evidence for charged soliton states in doped polyacetylene. Independent evidence has been obtained from the doping induced modes ${ }^{17,18}$ which appear in the mid-infrared associated with the local vibrational modes of the charged bond-alternation domain wall. Again, these mid-IR modes are independent of dopant ${ }^{19}$ (see also Figure 6) and show characteristic shifts after deuteration; ${ }^{18}(\mathrm{CD})_{x}$ compared to $(\mathrm{CH})_{x}$. That all of these spectroscopic features are associated with charged solitions has been unambiguously proven through a series of photoinduced absorption ${ }^{20,21}$ and photoinduced ESR measurements. ${ }^{22}$

The remarkable oscillator strength associated with these doping induced spectroscopic features arises directly from the spatial extent of the charge storage state. ${ }^{23}$ Whereas one might anticipate that the ratio of oscillator strengths would be equal to the dopant concentration, a detailed theoretical analysis shows that for dilute concentrations the mid-gap transition is enhanced by a factor of about (2l) where $(2 l a)$ is the full width of the bondalternation domain wall and $a$ is the carbon-carbon spacing along the $(\mathrm{CH})_{x}$ chain. Since $(2 l) \simeq 14$, this enhancement of the soliton transition makes it observable even at highly dilute dopant concentrations. A complete analysis of the strength of the mid-gap transition is included in the work of Feldblum et al ${ }^{15}$ who find the results to be in good quantitative agreement with theory.

\section{b) Photoinduced Absorption Studies of trans- $(\mathrm{CH})_{x}$}

Information on the intrinsic properties of the non-linear soliton excitations has been obtained from a series of photoinduced absorption experiments. These studies were stimulated by the calculations of $\mathrm{Su}$ and Schrieffer ${ }^{24}$ that showed solitons could be photogenerated. Their results demonstrated that in trans- $(\mathrm{CH})_{x}$ an $\mathrm{e}-\mathrm{h}$ pair should evolve into a pair of solitons within an optical phonon period, or about $10^{-13} \mathrm{~s}$. Photoinduced excitations have been observed and their dynamics have been studied. These experimental results on the photoinduced (PI) changes in absorption confirmed the predictions and established the reverse spin-charge relation of the soliton model.

The photogeneration of soliton-antisoliton pairs implies formation of states at mid-gap. Time resolved spectroscopy ${ }^{25}$ has been used to observe the predicted absorption due to photogenerated intrinsic gap states. Moreover, the time scale for photogeneration of these gap states has been investigated. ${ }^{26,27}$ Using sub-picosecond resolution, these studies demonstrated that the gap states and the associated interband bleaching are produced in less than $10^{-13} \mathrm{~s}$, consistent with the theoretical predictions.

Vardeny et al..$^{21}$ and Blanchet et al. ${ }^{20}$ have observed the photoinduced absorption arising from both the mid-gap electronic transition (sketched on 


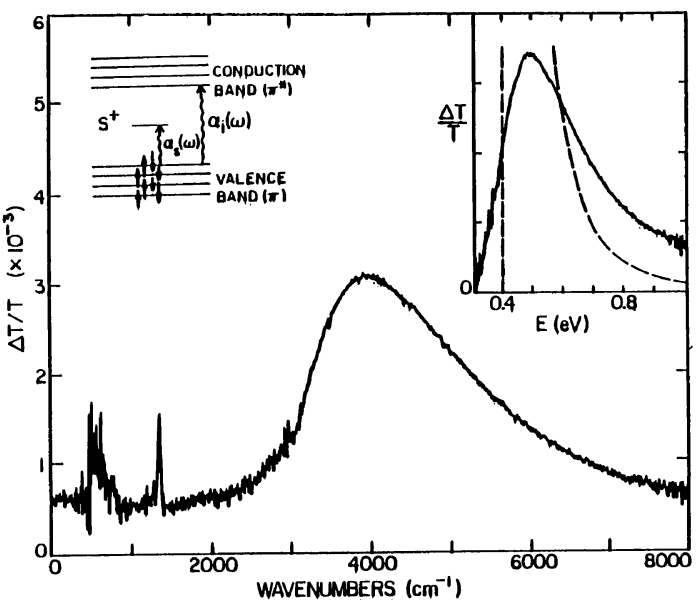

Figure 7. Photoinduced absorption in trans- $(\mathrm{CH})_{x}$ (see Blanchet, 1983a). The solitons are photogenerated using photons with $\hbar \omega>E_{\mathrm{g}}$. The subsequent photoinduced absorption process (which occurs at $\sim 0.5 \mathrm{eV}$ ) involving the mid-gap state for a positive soliton is sketched (left inset). A similar process (mid-gap to conduction band) occurs for the negative soliton. The infrared modes, 1370 and $1260 \mathrm{~cm}^{-1}$, provide information on the internal vibrational modes of the photogenerated domain walls. The $500 \mathrm{~cm}^{-1}$ peak is the "pinned" mode (see the text). The mid-gap transition is shown more clearly in the upper right; it has the characteristic asymmetry expected for the theoretical lineshape (dashed curve) (see Blanchet et $\left.a .^{20}\right)$.

Figure 2) and the associated infrared active modes introduced by the local lattice distortion. The results of Blanchet et al. ${ }^{20}$ are reproduced in Figure 7. The $0.5 \mathrm{eV}$ absorption is the mid-gap transition. The IR modes at $1370 \mathrm{~cm}^{-1}$ and $1260 \mathrm{~cm}^{-1}$ are consistent with the calculations of Mele and Rice (1980) and Horowitz (1980) which predict the appearance of IR active internal vibrational modes between each of the Raman modes of pure trans$(\mathrm{CH})_{x}$.

Infrared spectroscopy studies $^{17-19}$ of lightly doped trans- $(\mathrm{CH})_{x}$ have demonstrated that the same spectroscopic features arise upon doping. Moreover, these doping-induced absorptions are independent of the dopant and are therefore identified as intrinsic features of the doped trans- $(\mathrm{CH})_{x}$ chain. These important results demonstrate that both the photoinduced spectroscopic features and those induced by doping are associated with the same charged state. Moreover, the observed frequencies and lineshapes are consistent with those expected for charged soliton excitations. ${ }^{18,19}$ It has been argued $^{28}$ that these changes in infrared absorption are general features of charged localized states on the trans- $(\mathrm{CH})_{x}$ chain (solitons or polarons) and thus not definitive proof of the photogeneration of solitons. However, since these excitations have the reversed spin-charge relation of solitons, this ambiguity is settled (Flood et al. 1981, 1982).

\section{BIPOLARONS IN POLY(THIOPHENE): IN-SITU ABSORPTION STUDIES}

\section{a) Experimental Results}

Figure 8 shows a series of absorption spectra ${ }^{9}$ taken in-situ during the doping cycle at different applied voltages: $3.6 \mathrm{~V}(y=2.8 \%), 3.65 \mathrm{~V}(y=4 \%)$, $3.7 \mathrm{~V}(y=5.4 \%), 3.8 \mathrm{~V}(y=9.6 \%), 3.85 \mathrm{~V}(y=12 \%)$, $4.05 \mathrm{~V}(y=20 \%)$. In each case the cell was allowed to come to quasi-equilibrium before taking the spectra. Doping levels were obtained from direct electrochemical measurements for $V_{\text {app }} v s$. $A$ in a parallel experiment using Electrochemical Voltage Spectroscopy. ${ }^{9}$

As the doping proceeded via the oxidation re-

$$
\begin{aligned}
& {\left[\left\langle\mathrm{S}^{\prime}\right\rangle\right]_{x}+x y \mathrm{LiClO}_{4} \longrightarrow } \\
& {\left[\left[\left\langle{ }_{\mathrm{S}} \backslash\right]^{+y}\left(\mathrm{ClO}_{4}^{-}\right)_{y}\right]_{x}+x y \mathrm{Li}\right.}
\end{aligned}
$$

action, the intensity of the interband transition decreased continuously and the absorption peak shifted toward higher energy. In addition, the two new absorption features appeared in the IR below the gap edge with intensities which increased as the dopant level increased. The lower energy IR peak remains at a constant energy $(\sim 0.65 \mathrm{eV})$ while the higher energy one shifts toward higher energy as the dopant level is increased. At $4.3 \mathrm{~V}$, the frequency dependent absorption is characteristics of the free carrier spectrum of the metallic state, similar to that found in heavily doped (either chemically or electrochemically) polyacetylene. ${ }^{19}$

The different spectra of Figure 9 show that the oscillator strength which appears below the gap edge comes primarily from the interband transition. In contrast to trans-polyacetylene, ${ }^{15,23}$ the loss of interband oscillator strength is not uniform but is 


\section{A. J. HeEger}

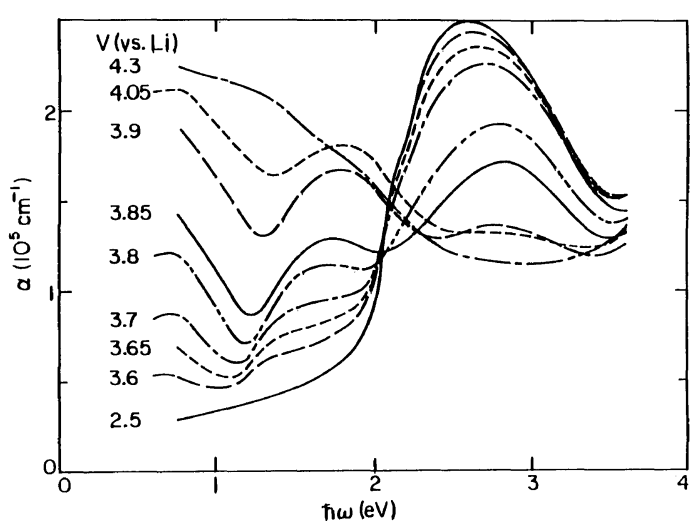

Figure 8. In-situ absorption curves for poly(thiophene) during electrochemical doping with $\left(\mathrm{ClO}_{4}\right)^{-}$. The applied voltages (vs. Li) are shown on the left. The corresponding concentrations were obtained EVS measurements and are as follows (in $\mathrm{mol} \%$ per thiophene ring): $3.60 \mathrm{~V}(y=2.8 \%) ; 3.65 \mathrm{~V}(y=4 \%) ; 3.70 \mathrm{~V}(y=$ $5.4 \%) ; 3.80 \mathrm{~V}(y=9.6 \%) ; 3.85 \mathrm{~V}(y=12 \%) ; 3.90 \mathrm{~V}(y=$ $14 \%) ; 4.05 \mathrm{~V}(y=20 \%)$; (see Chung et al..$\left.^{9}\right)$.

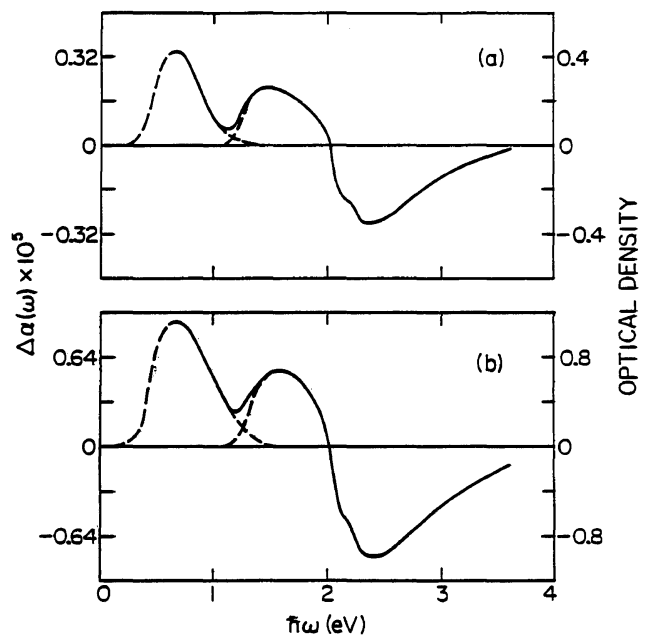

Figure 9. Difference spectra obtained from the data of Figure 9: a) $V_{\mathrm{app}}=3.65 \mathrm{~V}, y=4 \%$ (or $1 \%$ per carbon); b) $V_{\text {app }}=3.85 \mathrm{~V}, y=12 \%$ (or $3 \%$ per carbon). In each case the neutral point spectrum $\left(V_{\mathrm{app}}=2.50 \mathrm{~V}\right)$ was used as the reference. The dashed curves are extrapolations which attempt to separate the contributions from the two absorption peaks (see the text; see Chung et al. ${ }^{9}$ ).

greatest for frequencies near the band edge.

\section{b) Discussion in Terms of Confined S̄̄ Pairs or Bipolarons}

The absorption spectrum of neutral poly(thio- phene) is remarkably similar to that of trans$(\mathrm{CH})_{x},{ }^{15,23}$ but blue-shifted by about $0.4-0.5 \mathrm{eV}$. Even the familiar structure on the leading edge, attributed by Mele to dynamical chain distortion following electron-hole creation, ${ }^{29}$ is evident in the data. These similarities are suggestive that poly(thiophene) can be viewed as similar to trans- $(\mathrm{CH})_{x}$, but with the ground state degeneracy lifted by a small amount due to the inequivalence of the two structures with opposite bond alternation. This analogy was emphasized in the schematic structure shown in Figure 2.

Although this description of PT is admittedly schematic, there is experimental evidence that it represents an excellent starting point for a more detailed description of the doping processes in this system. The optical absorption data (Figure 9) indicate an energy level structure at dilute doping levels as shown in Figure $3 b$ with $\hbar \omega_{1}$ and $\hbar \omega_{2}$ just below the peaks since the transitions involved are between a localized gap state and the valency band density of states. The value for the interband absorption can be estimated from the data of Figures 8 and 9 to be $\hbar \omega_{1} \simeq 2.1 \mathrm{eV}$. In this case, the joint density of states of the valence and conduction bands is involved in the absorption so that the point of steepest slope in $\alpha(\omega)$ or the crossover in the difference curves (Figure 9) is used. Although there is surely some uncertainty in the assignment of the precise energies, the data from the dilute dopant concentrations yield:

$$
\hbar \omega_{1}+\hbar \omega_{2} \simeq \hbar \omega_{\mathrm{I}}=E_{\mathrm{g}}
$$

where $E_{\mathrm{g}} \equiv 2 \Delta_{0}$ is the energy gap. The results expressed quantitatively in eq 4 indicate the existence of electron-hole symmetry in the doped polymer. Referring to Figure $3 b$, the two doping induced energy levels appear symmetrically with respect to the gap center at $\pm \hbar \omega_{0}=0.40 \pm 0.05 \mathrm{eV}$. The existence of electron-hole symmetry implies that the schematic structure of Figure 4 represents an essentially correct point of view. The sulfur atoms stabilize the polyene chain in the configuration of Figure 4 through covalent bonding to neighboring carbon atoms. However, evidently the sulfur is only weakly interacting with the $\pi$-electron system of the polyene backbone. If this were not the case, some of the transferred charge would reside on the sulfur, and the electron-hole symmetry implied by Figures 8 and 9 and sketched in Figure $3 b$ would not be 
present.

We assign the two energy gap states inferred from Figures 8 and 9 to the two levels expected from charge storage in bipolaron states in doped PT. ${ }^{1,5,10-12}$ This assignment is based on three facts:

1) The two transitions imply formation of two levels symmetric with respect to the gap center.

2) The observation of only two transitions implies that the two levels are not occupied. If there were electrons in the lower level (as would be the case for a "hole" polaron) then a third absorption would be evident arising as a transition between the two localized levels. This is not observed.

3) Analysis of the data leads to $\left(\omega_{0} / \Delta_{0}\right) \simeq 0.35$. This small value is inconsistent with polaron formation for which $\left(\omega_{0} / \Delta_{0}\right) \geq 0.707 .^{5 a, b, c, 12}$

The small value inferred for $\left(\omega_{0} / \Delta_{0}\right)$ implies weak confinement. Using the results obtained by Fesser, Campbell, and Bishop ${ }^{12}$ in their detailed analysis of the bipolaron problem, we can extract values for the relevant microscopic parameters. The confinement parameter is defined as:

$$
\gamma=\Delta_{\mathrm{e}} / \lambda \Delta_{0}
$$

where $\Delta_{\mathrm{e}}$ is the constant external gap parameter $\left(2 \Delta_{\mathrm{e}}\right.$ would be the energy gap without the bond alternation contribution, $\Delta_{\mathrm{i}}$, which arises from the spontaneous symmetry breading due to the Peierls instability), $\lambda$ is the dimensionless electron-phonon coupling constant appropriate to the two-fold commensurate case, and $\Delta_{0}$ is the full gap parameter $\left(\Delta_{0}=\Delta_{\mathrm{e}}+\Delta_{\mathrm{i}}\right)$. From Figure 4 of $\mathrm{FBC},{ }^{12}$ we obtain $\gamma \simeq 0.1-0.2$ using the experimental value for $\left(\omega_{0} / \Delta_{0}\right) \simeq 0.35$. This small value for $\gamma$, implying weak confinement, is consistent with the point of view expressed in Figure 4 and with the remarkable similarity of the shape of the absorption curves of neutral trans- $(\mathrm{CH})_{x}$ and neutral PT.

In the heavily doped limit $\left(V_{\text {app }}=4.3 \mathrm{~V}\right)$, all signs of the interband transition have disappeared, and the spectrum (Figure 9) is dominated by the free carrier absorption in the infrared. In this regime, the optical properties of doped PT are those of a metal. The magnitude and spectral dependence of $\alpha(\omega)$ are similar to those reported earlier ${ }^{30}$ for $\mathrm{Na}$-doped trans $-(\mathrm{CH})_{x}$ where electrical conductivities in excess of $10^{3} \Omega^{-1}-\mathrm{cm}^{-1}$ were inferred from the frequency dependent absorption in the IR and subsequently observed directly in DC measurements. ${ }^{31}$ Thus, metallic doped PT can be expected to be an excel- lent conductor. Although previous conductivity measurements on electrochemically synthesized (and doped) PT have yielded values ${ }^{32}$ as high as $100 \Omega^{-1}-\mathrm{cm}^{-1}$, the intrinsic values may in fact be much higher.

\section{CONCLUSION}

Extensive studies of polyacetylene have demonstrated that the coupling of electronic excitations to nonlinear conformational changes is an intrinsic and important feature of conducting polymers. Although this coupling and the degenerate ground state lead to the novel soliton excitations in trans$(\mathrm{CH})_{x}$, generalization of these concepts and application to the larger class of conjugated polymers has been an obvious goal of the field. The experimental evidence of electron-hole symmetry and weak confinement in poly(thiophene) makes this polymer a nearly ideal example of a model system in which the ground state degeneracy has been lifted. The study of bipolarons (or confined charged solitons) in poly(thiophene) has demonstrated that the concepts carry over in detail and that a quantitative understanding of the resulting phenomena is possible even for relatively complex systems.

Acknowledgement. Supported by the Office of Naval Research.

\section{REFERENCES}

1. A. J. Heeger, Comments on Solid State Physics, 10, (1981).

2. a) W. P. Su, J. R. Schrieffer, and A. J. Heeger, Phys. Rev. Lett., 42, 1698 (1979).

b) W. P. Su, J. R. Schrieffer, and A. J. Heeger, Phys. Rev. B, 22, 2099 (1980).

3. M. J. Rice, Phys. Lett., 71A, 152 (1978).

4. H. Takayama, Y. R. Lin-Lin, and K. Maki, Phys. Rev. B, 21, 2388 (1980).

5. a) S. A. Brazovskii, JETP Lett., 28, 656 (1979).

b) S. A. Brazovskii, JETP Lett., 78, 677 (1980).

c) S. A. Brazovskii and N. Kirova, JETP Lett., 33, 4 (1981).

6. B. R. Weinberger, E. Ehrenfreund, A. J. Heeger, and A. G. MacDiarmid, J. Chem. Phys., 72, 4749 (1980) and references therein.

7. M. Nechtschein, F. Devreaux, R. L. Green, T. C. Clark, and G. B. Street, Phys. Rev. Lett., 44, 356 (1980) and references therein.

8. a) R. Jackiw and C. Rebbi, Phys. Rev., B, 13, 3398 (1978). 


\section{A. J. HEEGER}

b) R. Jackiw and J. R. Schrieffer, Nucl. Phys., B, 190, 253 (1981).

9. T. C. Chung, J. H. Kaufman, A. J. Heeger, and F. Wudl, Phys. Rev. B (July 15, 1984).

10. a) J. L. Brédas, R. R. Chance, and R. Silbey, Mol. Cryst. Liq. Cryst., 77, 319 (1981).

b) J. L. Brédas, B. Themans, J. M. Andre, R. R. Chance, D. S. Boudreaux, and R. Silbey, J. Phys. (Paris), 44, 373 (1983).

11. L. Lauchlan, S. Etamad, T. C. Chung, A. J. Heeger, and A. G. MacDiarmid, Phys. Rev. B, 24, 3701 (1981).

12. K. Fesser, A. R. Bishop, and D. K. Campbell, Phys. Rev. B, 27, 4804 (1983).

13. a) P. J. Nigrey, A. G. MacDiarmid, and A. J. Heeger, J. Chem. Soc., Chem. Commun., 594 (1979).

b) P. J. Nigrey, D. McInnes, D. P. Nairns, A. G. MacDiarmid, and A. J. Heeger, J. Electrochem. Soc., 128, 1651 (1981).

c) P. J. Nigrey, A. G. MacDiarmid, and A. J. Heeger, Proceeding of the International Conference on "Low-Dimensional Conductors," Boulder, Colorado, Mol. Cryst. Liq. Cryst., 83, 1341 (1982).

14. D. McInnes, M. R. Druy, P. J. Nigrey, D. P. Nairns, A. G. MacDiarmid, and A. J. Heeger, J. Chem. Soc., Chem. Commun., 317 (1981).

15. A. Feldblum, J. H. Kaufman, S. Etamad, A. J. Heeger, T. C. Chung, and A. G. MacDiarmid, Phys. Rev., B, 26, 815 (1982).

16. a) J. H. Kaufman, J. W. Kaufer, A. J. Heeger, R. Kaner, and A. G. MacDiarmid, Phys. Rev. B, 26, 4 (1982).

b) J. H. Kaufman, T. C. Chung, and A. J. Heeger, Solid State Commun., 47, 585 (1983).

c) J. H. Kaufman, T. C. Chung, and A. J. Heeger, J. Electro. Chem. Soc. (in press).

17. C. R. Fincher, M. Ozaki, A. J. Heeger, and A. G.
MacDiarmid, Phys. Rev., B, 19, 4140 (1979).

18. S. Etemad, A. Pron, A. J. Heeger, A. G. MacDiarmid, E. J. Mele, and M. J. Rice, Phys. Rev., B, 23, 5137 (1981).

19. S. Etemad, A. J. Heeger, and A. G. MacDiarmid, Ann. Rev. Phys. Chem., 33, 443 (1982) and references therein.

20. a) G. B. Blanchet, C. R. Fincher, T. C. Chung, and A. J. Heeger, Phys. Rev. Lett., 50, 1938 (1983).

b) G. B. Blanchet, C. R. Fincher, and A. J. Heeger, Phys. Rev. Lett., 51, 2132 (1983).

21. Z. Vardeny, J. Orenstein, and G. L. Baker, Phys. Rev. Lett., 50, 2032 (1983).

22. J. D. Flood and A. J. Heeger, Phys. Rev., B, 28, 2356 (1983).

23. N. Suzuki, M. Ozaki, S. Etamad, A. J. Heeger, and A. G. MacDiarmid, Phys. Rev. Lett., 45, 1209 (1980); erratum, Phys. Rev. Lett., 45, 1483 (1980).

24. W. P. Su and J. R. Shcrieffer, Proc. Natl. Acad. Sci., 77, 5626 (1980).

25. J. Orenstein and G. L. Baker, Phys. Rev. Lett., 49, 1043 (1982).

26. C. V. Shank, P. Yan, R. L. Fork, J. Orenstein, and G. L. Baker, Phys. Rev. Lett., 49, 1660 (1982).

27. Z. Vardeny, J. Strait, D. Moses, T. C. Chung, and A. J. Heeger, Phys. Rev. Lett., 49, 1657 (1982).

28. B. Horowitz, Solid State Commun., 41, 729 (1980).

29. E. J. Melé, Proceedings of the Los Alamos Workshop, Synth. Metals, 9 (No. 2), 207 (1984).

30. T. C. Chung, A. Feldblum, A. J. Heeger, and A. G. MacDiarmid, J. Chem. Phys., 47, 5504 (1981).

31. T. C. Chung, F. Moraes, J. D. Flood, and A. J. Heeger, Phys. Rev., B, Rapid Commun., 29, 2341 (1983).

32. K. Kaneto, Y. Kohno, K. Yoshino, and Y. Inuishi, J. Chem. Soc., Chem. Commun., 382 (1983). 